\title{
Erratum to: Comparison of extraction methods for the analysis of natural dyes in historical textiles by high-performance liquid chromatography
}

\section{Lemonia Valianou • Ioannis Karapanagiotis •} Yannis Chryssoulakis

Published online: 24 February 2010

(C) Springer-Verlag 2010

\section{Erratum to: Anal Bioanal Chem} DOI 10.1007/s00216-009-3137-6

There was a mistake in Table 1 concerning the absorbance maxima for Rubiadin. The correct values for
Rubiadin are: 243, 277, $411 \mathrm{~nm}$. Also, we note that the Absorbance maxima shown in Table 1 are given in $\mathrm{nm}$. The corrected Table is provided below. Our apologies to the readers.

Table 1 Dyes and colouring compounds investigated in the study. UV-Vis absorbance maxima were measured by HPLC-DAD according to the Experimental

\begin{tabular}{|c|c|c|c|c|}
\hline Dye & Dye class $[2,36]$ & Biological source & Main components & Absorbance maxima (nm) \\
\hline \multirow[t]{3}{*}{ Madder } & \multirow[t]{3}{*}{ Mordant dye } & \multirow[t]{3}{*}{ Rubia tinctorum $\mathrm{L}$. } & alizarin & $247,277,429$ \\
\hline & & & puruprin & $255,293,479$ \\
\hline & & & rubiadin & $243,277,411$ \\
\hline Cochineal & Mordant dye & Dactylopius coccus Costa & carminic acid & $275,309,493$ \\
\hline \multirow[t]{3}{*}{ Weld } & \multirow[t]{3}{*}{ Mordant dye } & \multirow[t]{3}{*}{ Reseda luteola $\mathrm{L}}$. & luteolin & $253,265,345$ \\
\hline & & & luteolin-7-O-glucoside & $253,265,345$ \\
\hline & & & apigenin & 267,335 \\
\hline \multirow[t]{2}{*}{ Young fustic } & \multirow[t]{2}{*}{ Mordant dye } & \multirow[t]{2}{*}{ Cotinus coggygria Scop. } & fisetin & $245,321,359$ \\
\hline & & & sulfuretin & $257,267,377,397$ \\
\hline \multirow[t]{3}{*}{ Turmeric } & \multirow[t]{3}{*}{ Direct dye } & \multirow[t]{3}{*}{ Curcuma longa $\mathrm{L}$. } & curcumin & 263,425 \\
\hline & & & demethoxycurcumin & 249,421 \\
\hline & & & bisdemethoxycurcumin & 247,417 \\
\hline \multirow[t]{2}{*}{ Woad } & \multirow[t]{2}{*}{ Vat dye } & \multirow[t]{2}{*}{ Isatis tinctoria $\mathrm{L}$. } & indigotin & $241,285,330,605$ \\
\hline & & & indirubin & $239,289,363,539$ \\
\hline
\end{tabular}

The online version of the original article can be found under http://dx. doi.org/10.1007/s00216-009-3137-6.

L. Valianou $\cdot$ I. Karapanagiotis $(\bowtie)$

Ormylia Art Diagnosis Center,

Ormylia 63071 Chalkidiki, Greece

e-mail: g.karapanagiotis@artdiagnosis.gr

L. Valianou $\cdot$ Y. Chryssoulakis

School of Chemical Engineering,

National Technical University of Athens,

Athens 15773, Greece 